\title{
Effect of Hydronephrosis on Survival in Advanced Stage Cervical Cancer
}

\author{
Mehmet Rıfat Goklu ${ }^{1}$, Kerem Doga Seckin ${ }^{2 *}$, Cihan Togrul ${ }^{3}$, Yasemin Goklu ${ }^{1}$, \\ Ali Emre Tahaoglu', Murat $\mathrm{Oz}^{4}$, Ibrahim Egemen Ertas ${ }^{5}$
}

\begin{abstract}
Background: Hydronephrosis is frequently encountered in advanced stage cervical cancers, and may be associated with mortality. In the present study, we aimed to demonstrate the effect of hydronephrosis on survival in patients with inoperable advanced stage cervical cancer. Materials and Methods: The study data were acquired by retrospective analysis of the patient records belonging to 165 women with FIGO (International Federation of Gynecology and Obstetrics) stage-IIIB or more advanced cervical cancer, which were not surgical candidates. Parameters including patient age, pathological diagnosis, disease stage, pelvic sidewall extension, presence of hydronephrosis and administration of chemoradiation were analyzed. Further, the effects of these variables on survival were assessed. $P$ values less than 0.05 were considered statistically significant. Results: The distribution of the study patients according to disease stage was as follows: $131(79.4 \%)$ had stage-IIIB, $18(10.9 \%)$ had stage-IVB and 16 (\% 9.7) patients had stage-IVA disease. Hydronephrosis was not evident in $91(55.2 \%)$ of these patients, whereas $41(24.8 \%)$ had unilateral and $33(20 \%)$ patients had bilateral hydronephrosis. When compared to mean survival in patients who did not have hydronephrosis, survival was significantly shortened in patients who had bilateral and unilateral hydronephrosis $(\mathbf{p}<\mathbf{0 . 0 5})$. There was no significant survival difference between patients with unilateral and bilateral hydronephrosis $(p>0.05)$. Although patient age, pathological type, pelvic involvement, and chemotherapy treatment rates were similar $(p>0.05)$, radiotherapy requirement rate and disease stage were significantly different among the study groups $(\mathbf{p}<0.05)$. Conclusions: Hydronephrosis was found to be a significant predictor of poor survival in patients with advanced stage cervical cancer, irrespective of unilateral or bilateral involvement. While waiting for future studies with larger sample sizes, we believe that the FIGO stages in advanced cervical cancer could further be stratified into subgroups according to presence or absence of hydronephrosis.
\end{abstract}

Keywords: Cervical cancer - hydronephrosis - stage - survival

Asian Pac J Cancer Prev, 16 (10), 4219-4222

\section{Introduction}

Cervical cancer is a preventable and curable disease. Worldwide, in women under 45 years of age, it is the $2^{\text {nd }}$ most common type of cancer, and 3rd most common cause of cancer related death, following breast and lung cancer (Espey et al., 2007). According to year 2008 statistics, cervical cancer comprises $9 \%(539,800)$ of all cancer diagnoses, and $8 \%(275,100)$ of all cancer related deaths (Miller et al., 2008).

In contrast with other gynecologic cancers, cervical cancer is staged clinically (Benedet et al., 2000; Pecorelli 2009). The International Federation of Gynecology and Obstetrics (FIGO) staging is preferred in these patients, as it is less resource intensive, is more sensitive in detection of locally advanced disease (tumor size, vaginal or parametrial involvement) and identification of patients that are poor surgical candidates (Jemal et al., 2011). Parametrial involvement is critical in these patients, as its presence is considered a contraindication for surgery.

Infiltration of the bladder and ureter by the tumor results in hydronephrosis, and consequently, a nonfunctional kidney. Obstructive uropathy is encountered in 14 to 44 percent of the cases with cervical cancer, and is a common cause of cervical cancer related mortality (Chao et al. 1998; Rose et al., 2010; Wit and Horenbas, 2014; Patel et al., 2015). Besides medical treatment, percutaneous nephrostomy and ureteral stent placement is commonly undertaken. However, these interventions have not proven useful in prolongation of survival (Lee and Jones 1994; Pradhan et al., 2011).

In the present study, we aimed to demonstrate the effect of hydronephrosis on survival in Turkish patients with inoperable advanced stage cervical cancer.

${ }^{1}$ Obstetrics and Gynecology Department, Diyarbakir Maternity Hospital, Diyarbakir, ${ }^{2}$ Kanuni Sultan Suleyman Education and Research Hospital, Istanbul, ${ }^{3}$ Corum Hitit University, Corum, ${ }^{4}$ Zekai Tahir Burak Women's Health and Research Hospital, Ankara, ${ }^{5}$ Tepecik Education and Research Hospital, Izmir, Turkey*For correspondence: doga_seckin@hotmail.com 


\section{Materials and Methods}

The study was performed at the Gynecologic Oncology Department of İzmir Tepecik Training and Research Hospital in İzmir, Turkey, following approval from the institutional review board. Patient charts of women with advanced cervical cancer that were regularly followed up at the present institution were retrospectively analyzed. The study population consisted of 165 women who were being actively followed-up or who had deceased during follow-up. All of the patients had stage IIIB or more advanced disease, and none of them had surgical treatment. Patients who had less advanced disease than stage IIIB, who did not attend to regular followup visits or who had surgical treatment were excluded from the study. Staging was routinely performed via examination under general anesthesia, and with biopsy. In particular, hydronephrosis was diagnosed via intravenous pyelography (IVP) and abdominal ultrasound (US). Pelvic

\section{Table 1. Clinical Features of the Study Patients}

\begin{tabular}{|c|c|c|}
\hline & $\mathrm{n}$ & percentage \\
\hline \multicolumn{3}{|l|}{ Age (years) } \\
\hline$\leq 65$ & 128 & $77.60 \%$ \\
\hline$<65$ & 37 & $22.40 \%$ \\
\hline \multicolumn{3}{|l|}{ Cell type } \\
\hline Adeno-carcinoma & 81 & $49.10 \%$ \\
\hline Adeno-squamous & 6 & $3.60 \%$ \\
\hline Squamous Cell Carcinoma & 78 & $47.30 \%$ \\
\hline \multicolumn{3}{|l|}{ Stage } \\
\hline Stage-III & 131 & $79.40 \%$ \\
\hline Stage-IVA & 16 & $9.70 \%$ \\
\hline Stage-IVB & 18 & $10.90 \%$ \\
\hline \multicolumn{3}{|l|}{ Hydronephrosis } \\
\hline None & 91 & $55.20 \%$ \\
\hline Unilateral & 41 & $24.80 \%$ \\
\hline Bilateral & 33 & $20.00 \%$ \\
\hline \multicolumn{3}{|l|}{ Chemotherapy } \\
\hline Treated & 127 & $77.00 \%$ \\
\hline Non-treated & 38 & $23.00 \%$ \\
\hline \multicolumn{3}{|l|}{ Radiotherapy } \\
\hline Received & 158 & $95.80 \%$ \\
\hline Not received & 7 & $4.20 \%$ \\
\hline \multicolumn{3}{|l|}{ Pelvic sidewall involvement } \\
\hline None & 17 & $10.30 \%$ \\
\hline Unilateral & 74 & $44.80 \%$ \\
\hline Bilateral & 74 & $44.80 \%$ \\
\hline
\end{tabular}

sidewall involvement was determined using computerized tomography (CT) and magnetic resonance imaging (MRI). In patients who were diagnosed with hydronephrosis, percutaneous nephrostomy or ureteral stenting was employed, as clinically indicated. Patient age, pathological diagnosis, stage, pelvic sidewall involvement, presence of hydronephrosis, interventions for treatment of hydronephrosis, radiotherapy (RT) and chemoterapy treatments and survival were analyzed. Considering disease stage and metastasis status, chemoradiation, external beam RT plus brachytherapy or chemotherapy alone was administered.

SPSS 20.0 computer software (Statistical Package for Social Sciences, Chigaco, IL, USA) was used for statistical analyses. For descriptive statistical analyses of the study data, mean, standard error, median, ratio and frequencies were expressed, where appropriate. Kaplan-Meier (Logrank) method was used for survival analysis. P values less than 0.05 were considered statistically significant.

\section{Results}

A total of 165 patients aged between 29 and 83 were included in the study. Clinicopathological data including patient age, stage, tumor histology, presence of hydronephrosis, administration of chemotherapy or radiotherapy, and pelvic sidewall extension status are presented in Table 1. According to disease stage and metastasis status, 121 patients $(73.3 \%)$ were treated with chemoradiation, 37 were treated with external beam RT and brachytherapy, and 7 (4.2\%) were treated with chemotherapy only.

The patients were grouped according to study parameters and were compared in terms of survival. There was no statistically significant survival difference between 37 patients $<65$ years of age [58.23 months$95 \% \mathrm{CI}(48.39-68.07)]$ and 128 patients $\geq 65$ years of age [47.56 months- 95\% CI (33.46-61.66)] ( $>>0.05)$. When compared according to pathological subtypes, mean survival in patients with adenocarcinoma $(n=81)[54.68$ months (43.74-65.62)], adeno-squamous carcinoma $(n=6)$ [32.17 months (12.39-51.94)] and squamous cell carcinoma $(n=78)$ [54.87 months (44.46-65.28)] did not have a statistically significant difference $(\mathrm{p}>0.05)$. Mean survival was significantly shorter in patients with stage

Table 2. Relationship between Hydronephrosis and Survival

\begin{tabular}{|c|c|c|c|c|c|}
\hline Hydronephrosis & Mean & $\begin{array}{l}\text { CI-95\% } \\
\text { Min-Max }\end{array}$ & Median & $\begin{array}{l}\text { CI-95\% } \\
\text { Min-Max }\end{array}$ & P value* \\
\hline None & 71.52 & $(58.24-84.81)$ & 48 & $(36.96-59.04)$ & \multirow{3}{*}{0.0001} \\
\hline Unilateral & 42.21 & $(32.14-52.27)$ & 35 & $(26.06-43.94)$ & \\
\hline Bilateral & 29.93 & $(21.80-38.05)$ & 24 & $(17.32-30.68)$ & \\
\hline
\end{tabular}

*Kaplan-Meier (Log-rank), CI: Confidence Interval

Table 3. Association between Hydronephrosis Treatment and Survival

\begin{tabular}{lccccc}
\hline Hydronephrosis & Mean & $\begin{array}{c}\text { CI-95\% } \\
\text { Min-Max }\end{array}$ & Median & $\begin{array}{c}\text { CI-95\% } \\
\text { Min-Max }\end{array}$ & P value* \\
\hline Received & 38.45 & $(27.25-49.65)$ & 26 & $(19.44-32.56)$ & 0.616 \\
Not-Received & 35.78 & $(27.48-44.07)$ & 26 & $(21.31-30.69)$ & 0.616 \\
\hline
\end{tabular}

*Kaplan-Meier (Log-rank), CI: Confidence Interval 


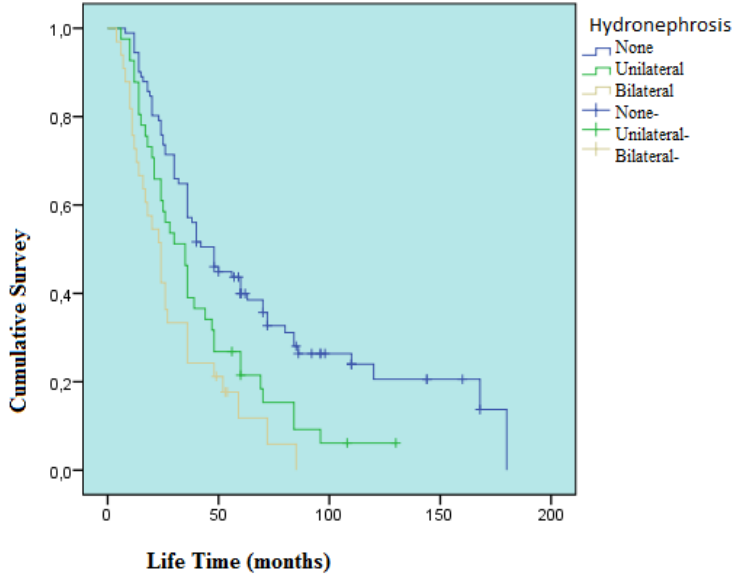

Figure 1. ROC Curve for Cumulative Survival and Hydronephrosis

IVA [22.13 months (17.33-26.92)] and stage IVB [33.89 months (22.53-45.24)] disease, when compared to patients with stage IIIB disease [63.53 months (53.27-73.79)] $(\mathrm{p}<0.05)$. Survival in patients with stage IVA and IVB disease did not have a statistically significant difference ( $p>0.05)$. Patients who were treated with chemotherapy [55.51 months (45.65-65.37)] were similar in terms of survival with patients who did not receive such treatment [60.94 months (44.06-77.81)] ( $\mathrm{p}>0.05)$. However, mean survival was significantly longer in patients that were treated with RT [58.53 months (49.50-67.57)] when compared to patients who did not receive RT [21.29 months (12.07-30.51)] $(\mathrm{p}<0.05)$.

Mean survival in patients with unilateral pelvic sidewall involvement [65.80 months (52.94-78.66)] was significantly longer than in patients with bilateral pelvic sidewall involvement [50.20 months (37.79-62.61)] and survival in patients without pelvic sidewall involvement [35.00 months $(18.44-51.56)](\mathrm{p}<0.05)$. Mean survival in patients with bilateral [29.93 months (21.80-38.05)] and unilateral hydronephrosis [42.21 months (32.1452.27)] was significantly shorter than in patients without hydronephrosis [71.52 months $(58.24-84.81)](\mathrm{p}<0.05)$ (Figure 1). Survival was similar in patients with unilateral and bilateral hydronephrosis ( $\mathrm{p}>0.05)$ (Table 2). Likewise, survival was similar in patients who had [38.45 months (27.25-49.65)] versus who did not [35.78 months (27.4844.07)] receive an intervention for the treatment of hydronephrosis $(\mathrm{p}>0,05)$ (Table 3$)$.

\section{Discussion}

Cervical cancer is a preventable disease. Early diagnosis and effective treatment is possible, thanks to various screening strategies (Demirtas, 2013). Primary intention should be prevention or early diagnosis, and screening programs constitute be the main focus. It is well known that the incidence of cervical cancer increases with age (Nartthanarung et al., 2014). In the present study, $78 \%$ of the patients were 65 years or older (Table 1). In advanced stage disease (peritoneal carcinomatosis, pulmonary metastasis-stage IVB), chemotherapy alone is recommended for treatment. Additional RT in this setting
DOI:http://dx.doi.org/10.7314/APJCP.2015.16.10.4219 Hydronephrosis and Survival in Advanced Cervical Cancer Cases is considered to carry risk for morbidity rather than to provide any survival benefit (Greer et al., 2010). Of the 18 study patients who had stage IVB disease, seven patients (47\%) were treated by chemotherapy only. According to our findings, it was concluded that only chemotherapy treatment did not have a significant impact on survival. In a meta-analysis that was published in 2010, it was demonstrated that chemoradiation was superior to RT only for treatment of locally advanced disease (Anon, 2010). In the present study, in accordance with the previous reports, it was found that approximately $87 \%$ of the patients were treated with chemoradiation, and these patients had longer mean survival than patients who were treated with RT only.

In previous studies, it was reported that there was discordance between clinical staging with gynecological examination and imaging modalities (CT, MRI) to determine the presence of parametrial and pelvic sidewall involvement. In studies by Hricak et al. and Mitchell et al., in locally advanced cervical cancers, imaging modalities were superior for staging purposes (Hricak et al., 2005; Mitchell et al., 2006; Shirazi et al., 2014). In contrast, clinical staging was found to be more effective in a study by Hancke et al (Hancke et al., 2008).

In the present study, unilateral or bilateral pelvic sidewall involvement was present in $89.6 \%$ of the study population, and the rate of hydronephrosis was $45 \%$. Pelvic sidewall involvement is not always associated with hydronephrosis. Seventy-four patients had unilateral $(44.8 \%)$ and 74 had bilateral $(44.8 \%)$ pelvic sidewall involvement. In 41 patients $(24.8 \%)$ there was unilateral hydronephrosis, whereas 33 (20\%) had bilateral hydronephrosis. Among 41 patients with unilateral hydronephrosis, 7 patients $(17 \%)$ did not have pelvic sidewall involvement, $16(39 \%)$ had unilateral pelvic sidewall involvement, and $18(44 \%)$ had bilateral pelvic sidewall involvement. Of the 33 patients with bilateral hydronephrosis, 8 patients $(24 \%)$ did not have pelvic sidewall involvement, whereas $4(12 \%)$ had unilateral pelvic sidewall involvement and 21 (64\%) had bilateral pelvic sidewall involvement. Consequently, pelvic sidewall involvement was assessed with clinical staging in this study, and some controversial results were demonstrated.

Disease stage is the most important prognostic variable in cervical cancer. Among 16 patients with stage IVA disease, 11 patients $(68.7 \%)$ had hydronephrosis (6 unilateral, 5 bilateral). On the other hand, among 18 stage IVB patients, 8 patients $(44.4 \%)$ had hydronephrosis $(5$ unilateral, 3 bilateral). These findings may indirectly reflect the negative effects of hydronephrosis on survival.

In a previous study by Pradhan et al., of the 143 patients with cervical cancer, $39(27 \%)$ had unilateral and $31(21.6 \%)$ had bilateral hydronephrosis. Survival was significantly shorter in patients with hydronephrosis (Pradhan et al., 2011). Likewise, our present study also demonstrated that hydronephrosis was associated with less survival. However, we did not observe a significant survival difference between patients with unilateral and bilateral hydronephrosis. As a result, it was concluded that hydronephrosis had unfavorable effects on survival, irrespective of laterality. Furthermore, the present study 
Mehmet Rifat Goklu et al

showed that interventions to treat hydronephrosis did not have a significant effect on survival. This finding may be due to not also heterogeneity in stage in the group of patients that received a particular treatment method, but also the timing of the treatment. The treatment groups were significantly heterogeneous for stage; of the 18 patients that were treated with double $\mathrm{J}$ catheter placement, 14 (77.7\%) had stage IIIB, $1(5.5 \%)$ had stage IVA and 3 $(16.5 \%)$ had stage IVB disease, and among the 10 patients who were treated with percutaneous nephrostomy, 7 (70\%) had stage IIIB, 2 (20\%) had IVA, 1 (10\%) had stage IVB disease. Thus, it is highly recommended to determine the patients with hydronephrosis during initial staging workup and to initiate appropriate treatment.

In summary, as an important cause of mortality due to cervical cancer, urinary system involvement and hydronephrosis has significant deleterious effects on survival. Failure to implement early treatment also may lead to decreased survival. Pelvic sidewall involvement is not always associated with hydronephrosis; however, it places the patients in stage IIIB according to FIGO staging system. Among the patients with stage IIIB tumors, although the stage is not altered in the presence of hydronephrosis, survival is significantly shortened, nearly approaching survival durations of patients with stage IV disease. In light of these findings, we recommend that stage IIIB tumors should further be stratified into subgroups according to presence of hydronephrosis.

\section{References}

Anon (2010). Reducing uncertainties about the effects of chemoradiotherapy for cervical cancer: individual patient data meta-analysis. Cochrane Database Syst Rev, 1, 82-5.

Benedet JL, Bender H, Jones H, et al (2000). FIGO staging classifications and clinical practice guidelines in the management of gynecologic cancers. Int J Gynaecol Obstet, 70, 209-62.

Chao KS, Leung WM, Grigsby PW, et al (1998). The clinical implications of hydronephrosis and the level of ureteral obstruction in stage IIIB cervical cancer. Int J Radiat Oncol Biol Phys, 40, 1095-100.

Demirtas B (2013). Review of strategies in promoting attendance for cervical screening. Asian Pac J Cancer Prev, 14, 3263-7.

Espey DK, Wu X-C, Swan J, et al (2007). Annual report to the nation on the status of cancer, 1975-2004, featuring cancer in American Indians and Alaska Natives. Cancer, 110,2119-52.

Greer BE, Koh W-J, Abu-Rustum NR, et al (2010). Cervical cancer. J National Compr Cancer Network, 8, 1388-416.

Hancke K, Heilmann V, Straka P, et al (2008). Pretreatment staging of cervical cancer: is imaging better than palpation?: Role of CT and MRI in preoperative staging of cervical cancer: single institution results for 255 patients. Ann Sur Oncol, 15, 2856-61.

Hricak H, Gatsonis C, Chi DS, et al (2005). Role of imaging in pretreatment evaluation of early invasive cervical cancer: results of the intergroup study American college of radiology imaging network 6651-gynecologic oncology group 183.J clin oncol, 23, 9329-37.

Jemal A, Bray F, Center MM, et al (2011). Global cancer statistics. CA Cancer J Clin, 61, 69-90.

Lee SK and Jones HW (1994). Prognostic significance of ureteral obstruction in primary cervical cancer. Int J Gynaecol Obstet, 44, 59-65.
Miller BA, Chu KC, Hankey BF and Ries LAG (2008). Cancer incidence and mortality patterns among specific Asian and Pacific Islander populations in the U.S. Cancer Causes Control, 19, 227-56.

Mitchell DG, Snyder B, Coakley F, et al (2006). Early invasive cervical cancer: tumor delineation by magnetic resonance imaging, computed tomography, and clinical examination, verified by pathologic results, in the ACRIN 6651/GOG 183 Intergroup Study. J Clin Oncol, 24, 5687-94.

Nartthanarung A, Thanapprapasr K, Udomsubpayakul U and Thanapprapasr D (2014). Age and survival of cervical cancer patients with bone metastasis. Asian Pac J Cancer Prev, 15, 8401-4.

Patel K, Foster NR, Kumar A, et al (2015). Hydronephrosis in patients with cervical cancer: an assessment of morbidity and survival. Support Care Cancer, 23, 1303-9.

Pecorelli S (2009). Revised FIGO staging for carcinoma of the vulva, cervix, and endometrium. Int J Gynaecol Obstet, 105, 103-4.

Pradhan TS, Duan H, Katsoulakis E, et al (2011). Hydronephrosis as a prognostic indicator of survival in advanced cervix cancer. Int J Gynecol Cancer, 21, 1091-6.

Rose PG, Ali S, Whitney CW, et al (2010). Impact of hydronephrosis on outcome of stage IIIB cervical cancer patients with disease limited to the pelvis, treated with radiation and concurrent chemotherapy: a Gynecologic Oncology Group study. Gynecol Oncol, 117, 270-5.

Shirazi AS, Razi T, Cheraghi F, et al (2014). Diagnostic accuracy of magnetic resonance imaging versus clinical staging in cervical cancer. Asian Pac J Cancer Prev, 15, 5729-32.

Wit EM, Horenblas S (2014). Urological complications after treatment of cervical cancer. Nat Rev Urol, 11, 110-7. 